\title{
Intercellular and intracellular functions of ceramides and their metabolites in skin (Review)
}

\author{
HWA JUN CHA ${ }^{1}$, CONGFEN HE $^{2}$, HUA ZHAO $^{2}$, YINMAO DONG $^{2}$, IN-SOOK AN $^{3}$ and SUNGKWAN AN ${ }^{1}$ \\ ${ }^{1}$ Korea Institute for Skin and Clinical Sciences, Konkuk University, Seoul 143-701, Republic of Korea; \\ ${ }^{2}$ Beijing Key Laboratory of Plant Resources Research and Development, China Cosmetic Research Center, \\ Beijing Technology and Business University, Beijing 100048, P.R. China; ${ }^{3}$ GeneCellPharm Incorporated, \\ Cheongju-si, Chungcheongbuk-do 361-951, Republic of Korea
}

Received September 17, 2015; Accepted May 6, 2016

DOI: $10.3892 / \mathrm{ijmm} .2016 .2600$

\begin{abstract}
The skin consists of the epidermis, dermis and subcutis. The epidermis is primarily comprised of keratinocytes and is separated into four layers according to the stage of differentiation of the keratinocytes. Corneocytes are terminally differentiated keratinocytes that closely interact with other corneocytes through corneodesmosomes, and synthesize lamellar bodies and the intercellular multilamellar barrier, which protects the body from the external environment. As ceramides are the principal components of lamellar bodies and the multilamellar barrier, it is important to understand the biosynthesis of ceramides and their functions in skin. Ceramides are synthesized by amide bond-mediated interactions between sphingoid bases, long-chain amino alcohols [long-chain base] and fatty acids through a de novo pathway, a sphingomyelin (SM) hydrolysis pathway and a catabolic pathway. The majority of ceramides produced by the de novo pathway form the epidermal barrier. Ceramides used as signaling molecules are synthesized by the SM and catabolic pathways. Synthesized ceramides are released from corneocytes and form the multilamellar barrier. Additionally, ceramides and their metabolites regulate the apoptosis, proliferation and differentiation of skin cells as well as the formation of the skin barrier. Thus, the study of ceramides and their metabolites is crucial to understanding the function and regulation of the skin barrier.
\end{abstract}

\section{Contents}

1. Introduction

2. Biosynthesis and structure of ceramides and their derivatives

3. Intracellular and extracellular functions of ceramides

4. Conclusion

Correspondence to: Dr Sungkwan An, Korea Institute for Skin and Clinical Sciences, Konkuk University, 120 Neungdong-ro, Gwangjin-gu, Seoul 143-701, Republic of Korea

E-mail: ansfgrc@konkuk.ac.kr

Key words: ceramide, skin, multilamellar barrier, ceramide metabolites

\section{Introduction}

Histologically, the skin consists of the epidermis, dermis and subcutis. The epidermis, which is the outermost layer of the skin, protects the human body from the external environment $(1,2)$. The epidermis is divided into four layers (stratum basale, stratum spinosum, stratum granulosum and stratum corneum) as a result of the process of epidermal differentiation. This creates an epidermal barrier at the level of the stratum corneum, the uppermost layer, to prevent dehydration and moisture loss. The epidermis also prevents external antigens from entering the skin and is a defense against ultraviolet (UV) rays (3-6). The epidermal barrier plays important roles in skin aging, dermatitis, psoriasis and atopic dermatitis, and is the subject of intense research (7-10).

Generally, the epidermal barrier is formed by the multiple actions of lipids produced in the lamellar bodies of the stratum granulosum during the process of keratinocyte differentiation, which involves terminally differentiated corneocytes and corneodesmosomes that connect keratinocytes $(11,12)$. These lipids create a multilamellar barrier between corneocytes, both increasing the adhesion and hindering the movement of material between cells, thus creating an epidermal barrier. The major lipids that form the multilamellar barrier of the skin consist of $50 \%$ ceramide, $25 \%$ cholesterol and $15 \%$ fatty acids (FAs) (11). Ceramides, also known to act as moderators of cellular physiology, are sphingolipids which are composed of FAs connected to sphingosine $(12,13)$.

\section{Biosynthesis and structure of ceramides and their derivatives}

Ceramides are primarily synthesized in the endoplasmic reticulum (ER) of the stratum spinosum within the epidermis. They are transferred out of cells through lamellar bodies created in the stratum granulosum and create a multilamellar barrier between the corneocytes of the stratum corneum (14-18). Ceramides are chemically composed of a sphingoid base, which is a long-chain amino alcohol [long-chain base (LCB)], and a FA joined by an amide bond (Fig. 1). The sphingoid base may consist of dihydrosphingosine $(\mathrm{dS})$, sphingosine $(\mathrm{S})$, phytosphingosine $(\mathrm{P})$ 


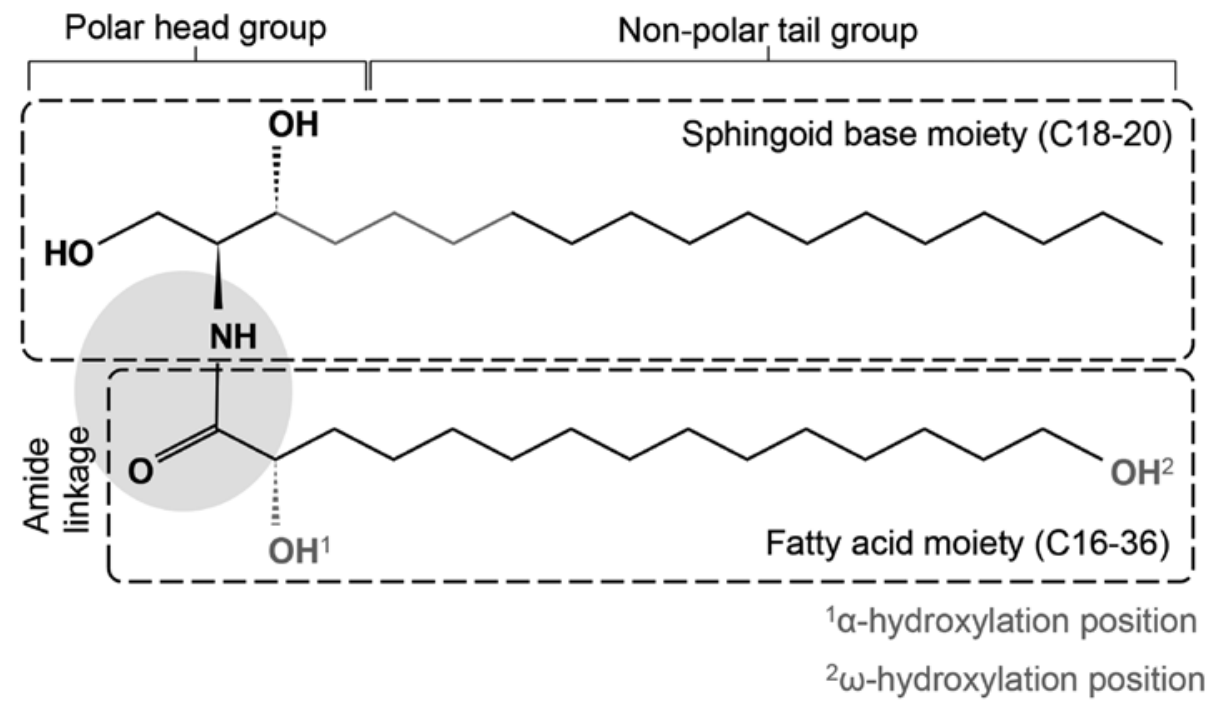

Figure 1. Basic chemical structure of ceramides. Ceramides are composed of a sphingoid base and a fatty acid (FA). The sphingoid base contains a polar head group and a non-polar tail group. Each moiety is bonded by an amide linkage. Additionally, the FA contains positions for $\alpha$ - and $\omega$-hydroxylation.

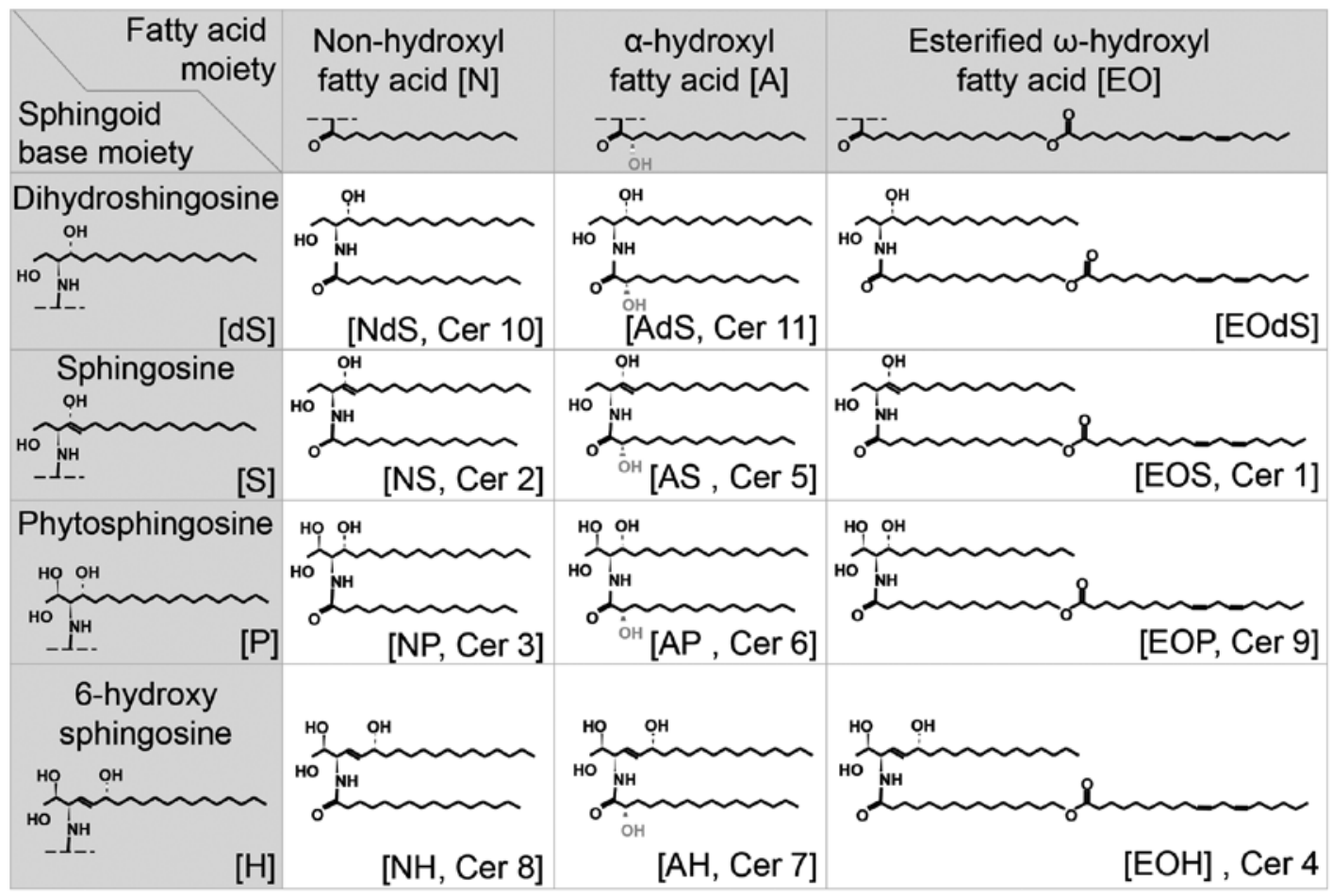

Figure 2. Details of de novo-generated ceramides in human stratum corneum. Various ceramides generated by the de novo pathway are formed by an amide linkage between the sphingoid base and fatty acid. The sphingoid base may consist of dihydrosphingosine (dS), sphingosine (S), phytosphingosine (P), or 6-hydroxy sphingosine (H). The fatty acid (FA) moieties may be a non-hydroxyl with FA (N), $\alpha$-hydroxyl fatty acid (A), or esterified $\omega$-hydroxyl fatty acid (EO). Cer, ceramide.

or 6-hydroxy sphingosine $(\mathrm{H})(19,20)$. The FA may be a non-hydroxyl FA (N), an $\alpha$-hydroxyl FA (A), or an esterified $\omega$-hydroxyl FA (EO). Thus, various ceramides are created by different combinations of these two types of molecules (Fig. 2). Ceramides undergo biosynthesis through various mechanisms, and the most common synthetic pathway is the de novo pathway, which is the most important biosynthetic mechanism for creating an epidermal barrier (Fig. 3). The de novo pathway can be divided into pathways that produce the sphingoid base and the FA.
The first step of the pathway responsible for sphingoid base synthesis uses palmitoyl-CoA and L-serine $(17,18)$. Initially, 3-ketosphinganine (ketodihydrosphingosine) reacts with palmitoyl-CoA and L-serine by serine palmitoyl transferase. The synthesized 3-ketosphinganine is deoxygenated by 3 -ketosphinganine reductase, producing sphinganine (dihydrosphingosine) $(17,18)$. The resulting long-chain amino alcohol of sphinganine has 18 carbon atoms; however, ceramides with $12-28$ carbon atoms have been found in the stratum corneum (21-24). 
Fatty acids

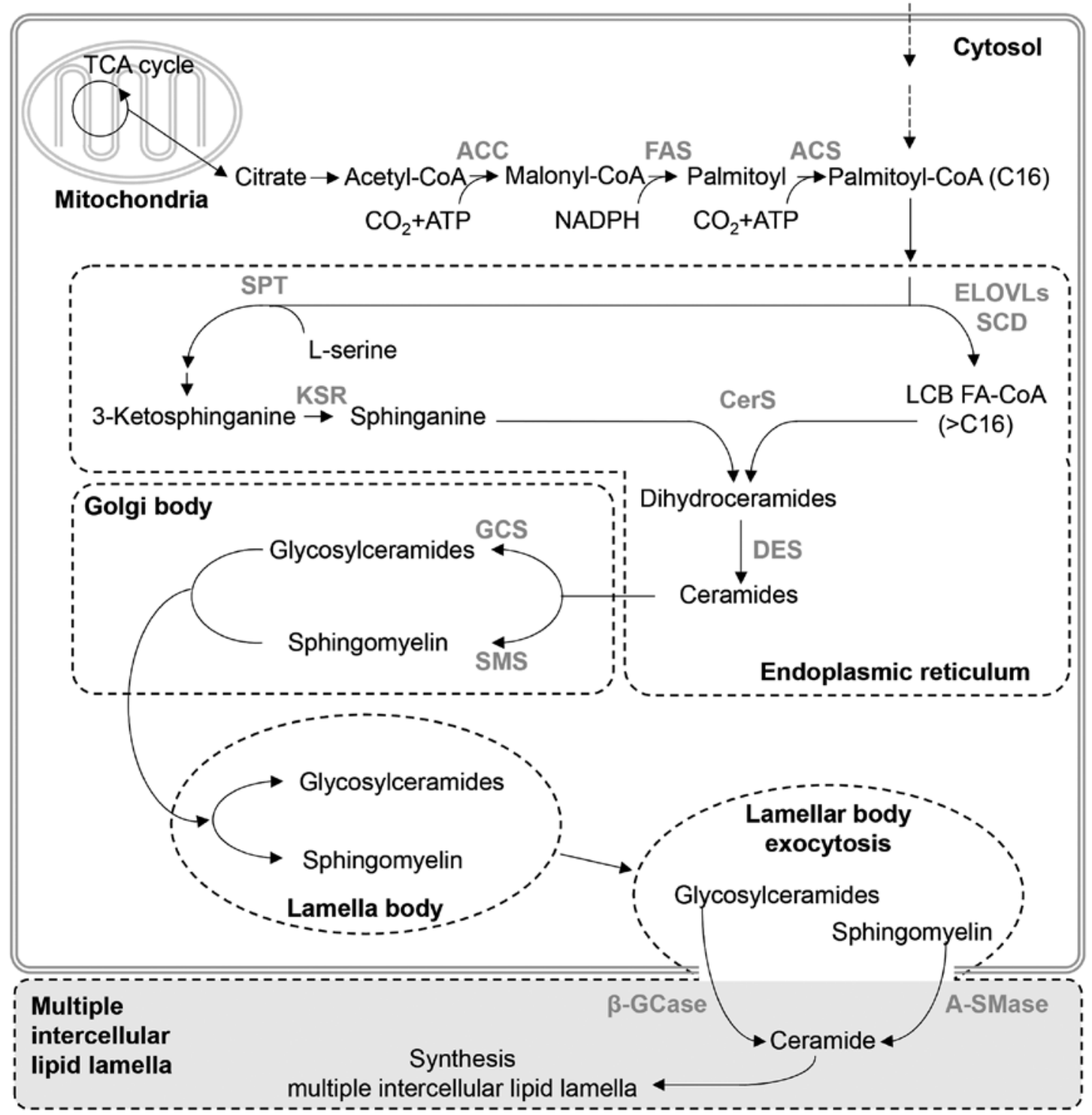

Figure 3. Synthesis and transfer of ceramides in the de novo pathway. The diagram shows that ceramides generated by the de novo pathway are contained in the ER. In the ER, serine palmitoyl transferase (SPT) and 3-ketosphinganine reductase (KSR) synthesize sphinganine. Additionally, elongation of very long chain fatty acids (ELOVL) protein and stearoyl-CoA desaturase (SCD) synthesize long-chain base (LCB) fatty acid-CoA (LCB FA-CoA). Dihydroceramide is produced by reacting sphingosine and LCB FA-CoA ceramide using ceramide synthase (CerS). From the ER, the generated ceramides exit the cell via the Golgi body. In this mechanism, ceramides are converted into glycosylceramide and sphingomyelin by glucosylceramide synthase (GCS) and sphingomyelin synthase (SMS). Once outside the cell, reconversion into ceramide occurs by $\beta$-glucocerebrosidase ( $\beta$-GCase) and acid sphingomyelinase (A-SMase). DES, dihydroceramide $\Delta 4$-desaturase; ACC, acetyl-CoA carboxylase; FAS, FA synthase; ACS, acyl-CoA synthetase.

Using acetyl-CoA carboxylase, FA synthase, and acyl-CoA synthetase, the FA synthesis pathway combines acetyl-CoA from citrate in the TCA cycle, malonyl-CoA and palmitoyl to synthesize palmitoyl-CoA with 16 carbon atoms. Then, after a condensation reaction by 3-ketoacyl-CoA synthase [elongation of very long chain FAs (ELOVL) protein] of palmitoyl-CoA, a reduction reaction by 3-keto-acyl-CoA reductase, a dehydration reaction by 3-hydroxyacyl-CoA dehydratase and a reduction reaction by 2,3-enoyl-CoA reductase, the carbon number of the FA is increased by 2 . Therefore, the length and saturation of the FA are determined by ELOVL proteins. For example, ELOVL6 creates C16 and C16:1, ELOVL1 creates C18-C24, ELOVL4 creates C24 or above, ELOVL3 creates C18-C24 and C18:1-C24:1, ELOVL7 creates C18-C22, ELOVL5 creates polyunsaturated C18-C20, and ELOVL2 creates polyunsaturated C20-C24 FAs $(25,26)$. In particular, ELOVL1, 3 and 4 are principally found in the epidermis $(27,28)$. The FAs subjected to long-chain elongation undergo hydroxylation at the $\alpha$ - or $\omega$-position, and the $\omega$-hydroxylation of FAs involves $\omega$-esterification with linoleic acid to produce ultra-long chain (ULC) FAs that have 28-38 carbon atoms $(14,29)$. Aside from creating ULC-ceramides, the $\omega$-hydroxyl group also connects proteins and ceramides through $\omega$-esterification to the side chain of glutamate in cornified envelope protein (30). Moreover, 1-O-acylceramides, in which very long-chain acyl residues are connected to the $\mathrm{N}$ - and $\mathrm{O}$-positions of ceramide, have also been discovered (31). 
The sphingosine base and FA are combined to produce dihydroceramides by $\mathrm{N}$-acylation which is catalyzed by ceramide synthase (CerS)1-6 (32). Finally, C4 and C5 are unsaturated by dihydroceramide $\Delta 4$-desaturase, creating ceramides (16-18). There are six types of CerS (CerS1 to CerS6) that produce different types of ceramides $(21,33)$. CerS3 and CerS4 are highly concentrated in the skin. CerS3 levels are elevated during keratinocyte differentiation and it has been found to be mutated in congenital ichthyosis $(34,35)$ Moreover, alopecia occurs in mice as a result of a lack of CerS4 (36). Therefore, CerS3 and CerS4 are expected to play significant roles in creating an epidermal barrier in human skin. Accordingly, NP (ceramide 3) and EOH (ceramide 4), created by CerS3 and CerS4, and long-chain ceramides with 18-26 carbons are known to be the major components of the epidermal barrier $(29,33,37)$. The amount of total lipids in the stratum corneum is low in patients with atopy and dry skin, and ceramide levels are also low $(38,39)$. Decreases in EOS (ceramide 1) levels are known to convert the orthorhombic structure of the epidermal barrier to a hexagonal gel structure, thus increasing moisture loss from the skin (40-42). The ceramides known to play important roles in the lamellar structure of the skin are EOS, NP and EOH, among which EOS is known to be an essential component in creating the lamellar structure (14). Ceramides produced in the ER are converted into glucosylceramides and sphingomyelin (SM) by glucosylceramide synthase and SM synthase (SMS), respectively, and are translocated to the Golgi complex to create the lamellar body (43). These compounds then exit the cell and are converted back into ceramides by $\beta$-glucocerebrosidase and acid sphingomyelinase (A-SMase), creating the multilamellar barrier.

Aside from the biosynthetic mechanism through which ceramides are produced, ceramides and their derivatives are synthesized by the SM and catabolic pathways (44) and used as intracellular messengers. The SM pathway synthesizes ceramides through the hydrolysis of SM by sphingomyelinase (SMase), and the typical SMases which play a role in this mechanism are epidermal A-SMase and neutral SMase $(45,46)$. By contrast, to synthesize SM, SMS uses ceramide. Moreover, the catabolic pathway uses ceramidase to produce derivatives of sphingosine and sphingosine-1-phosphate (S1P) to produce sphingosine from ceramide, and synthesizes ceramides from sphingosine in the reverse direction through CerS (44). Moreover, S1P is created when sphingosine is phosphorylated by sphingosine kinase, and sphingosine may be regenerated when S1P is dephosphorylated by S1P phosphatase. Ceramides and their derivatives act as different cellular messengers, which are repeatedly synthesized and degraded through reversible processes by multiple enzymes.

\section{Intracellular and extracellular functions of ceramides}

Ceramides and their derivatives act as intra- and extracellular messengers in the epidermal barrier $(9,12)$. Lipids that form the multiple intercellular lipid lamellae may be used to illustrate the structure of the epidermal barrier, either by a two compartment model or a bricks and mortar model (Fig. 4).

With regard to the detailed lipid structure and layout of multiple intercellular lipid lamellae, the structure of lipids in the stratum corneum was analyzed by X-ray diffraction in the 1950s and 1960s, and the structure of lamellae was determined by electron microscopy in the 1960s $(47,48)$. Structural analysis revealed that the lamellar structure had unique $13-\mathrm{nm}$ intervals (long periodicity phase), which disappeared when the temperature rose above $70^{\circ} \mathrm{C}$ and was regained at temperatures below $25^{\circ} \mathrm{C}$, proving that the structure is reversible (49-51). A structure of 6-nm intervals (short periodicity phase) was observed in certain types of ceramides (52). Moreover, lamellae have three different structures according to the layout of the head group and the packing of the alkyl group: orthorhombic, hexagonal gel and liquid lamellar (53). As a result of analyzing wide-angle $\mathrm{X}$-ray diffraction results, these structures show only the peak orthorhombic patterns of 0.375 and $0.416 \mathrm{~nm}$, and the peak liquid lamellar pattern of $0.46 \mathrm{~nm}$. At $45^{\circ} \mathrm{C}$, the peak orthorhombic and liquid lamellar patterns disappear, and only the $0.412 \mathrm{~nm}$ peak hexagonal gel pattern appears, which becomes one phase when the temperature rises $(52,53)$. If the temperature rises to $70^{\circ} \mathrm{C}$ and the motility of the alkyl chain increases, the peak liquid lamellar pattern of $0.46 \mathrm{~nm}$ is observed (39). The epidermal barrier structure consists of liquid lamellar < hexagonal gel $<$ orthorhombic, according to structural differences.

Intracellular ceramides act as second messengers for various processes (apoptosis, cell growth, differentiation, senescence, diabetes, insulin resistance, inflammation, neurodegenerative disorders or atherosclerosis) (54-57). Ceramides which play roles in intracellular signal transduction are produced using the aforementioned de novo pathway, which participates in different reactions according to the specific isoforms and activity of CerS (58). Most ceramides induce cellular apoptosis or growth arrest. For example, C18-ceramide is created by CerS1, which induces cell growth inhibition and apoptosis. However, there is an exceptional case in which the activity of CerS6 increases, rescuing the cells from ER stress and apoptosis by creating acyl-C16-ceramide (59).

One synthetic mechanism produces ceramides through the hydrolysis of SM (Fig. 5). Six types of SMases have been discovered in mammals: four types of neutral SMases, one A-SMase, and one alkaline SMase. Ceramides used in intracellular signal transduction are primarily created by neutral SMases existing in the ER and plasma membrane (60). Another mechanism synthesizes ceramides from S1P using S-1-phosphate phosphatase and CerS. An additional mechanism produces ceramides through the phosphorylation of ceramide-1-phosphate phosphatase (CPP) generated from ceramide-1-phosphate. Most ceramides created in this way inhibit cell growth and induce apoptosis (61-64). Thus, intracellular ceramides are known to be increased by inducing apoptosis through TNF- $\alpha$, Fas, radiation, and antitumor agents, and by the conversion of SM into ceramides in cell membranes or lysosomes. Moreover, ceramides are known to induce apoptosis by activating intracellular c-Jun N-terminal kinase (JNK)/stress-activated protein kinase (SAPK), protein kinase $\mathrm{C}(\mathrm{PKC}) \delta / \varepsilon$ and caspase-3 (CASP3)-like protease signals, and to reduce the phosphorylation of Ser473 of AKT by activating $\mathrm{PKC} \zeta$ and $\mathrm{PP} 2 \mathrm{~A}$, thus inhibiting cell growth $(65,66)$. Multiple mechanisms exist as the long carbon chain of ceramides make it structurally difficult for ceramides to pass through cell membranes. Thus, there is 

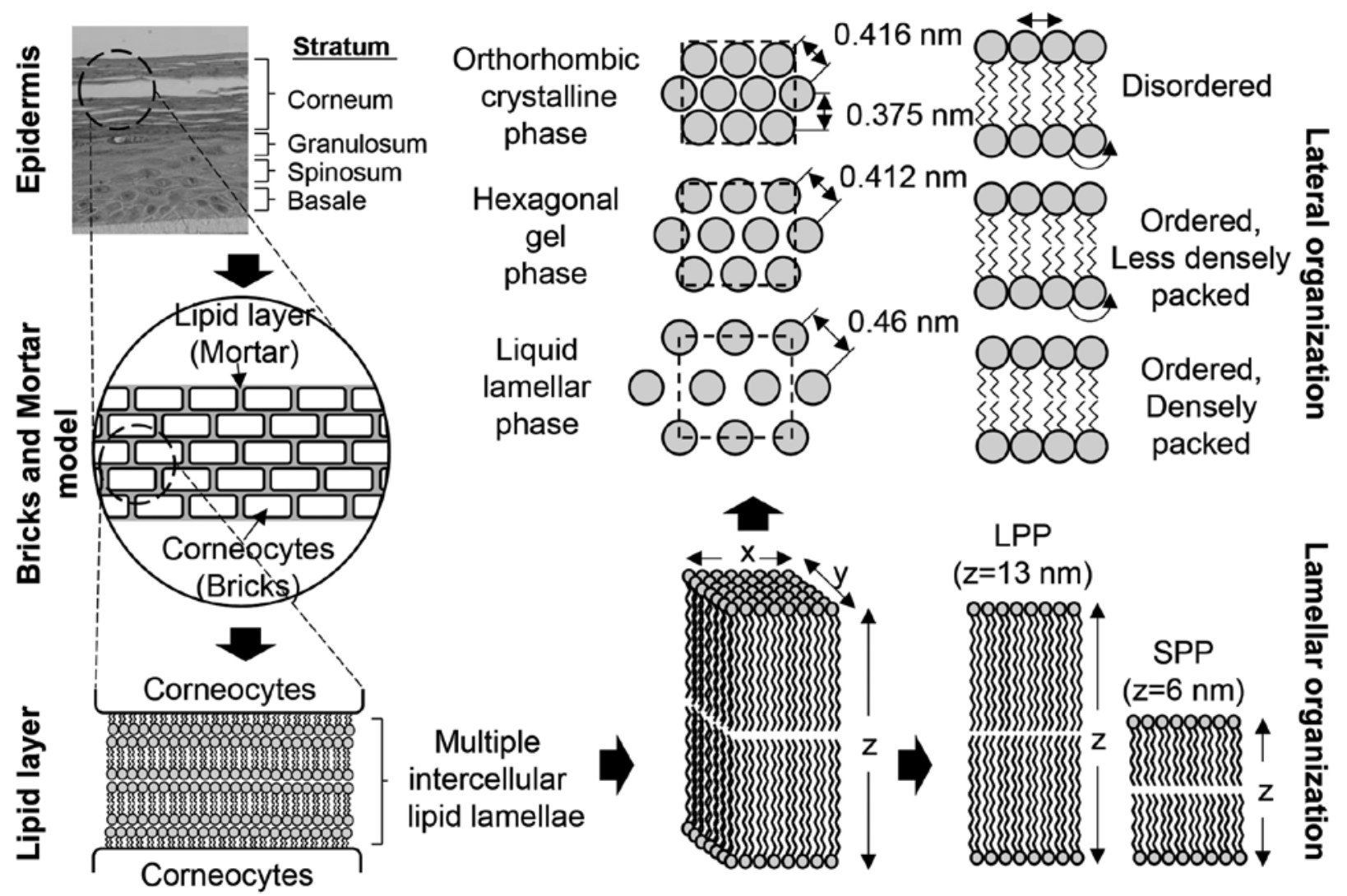

Figure 4. Structure of the multiple lamellae. The stratum corneum is formed by corneocytes (bricks) and lipids (mortar). The lipid layer is packed with intercellular spaces and multiple intercellular lamellae. The multiple lamellae have both lateral and lamellar levels of organization. The lateral organization is categorized as orthorhombic crystalline, hexagonal gel, and liquid lamellar, according to the temperature. The lamellar organization is categorized as long periodicity phase (LPP) and short periodicity phase (SPP), according to the type of ceramide.

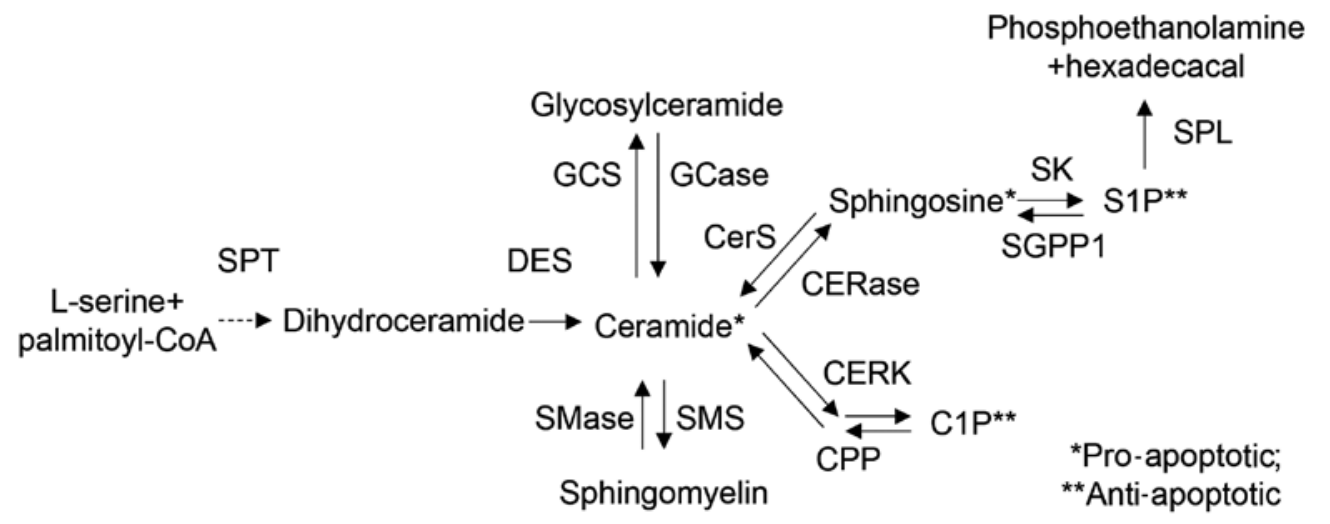

Figure 5. Overview of sphingolipid metabolism and related enzymes. Ceramides are synthesized from L-serine and palmitoyl-CoA by serine palmitoyl transferase (SPT). The generated ceramides are metabolized into glycosylceramide, sphingomyelin, sphingosine, sphingosine-1-phosphate (S1P), and ceramide-1-phosphate (C1P) by various enzymes, such as ceramide synthase (CerS), dihydroceramide $\triangle 4$-desaturase (DES), ceramide kinase (CERK), ceramide-1-phosphate phosphatase (CPP), sphingomyelin synthase (SMS), sphingomyelinase (SMase), ceramidase (CERase), sphingosine kinase (SK), S1P phosphatase (SGPP1), and S1P lyase (SPL). Each metabolite functions as a signaling molecule in apoptosis, cell growth, differentiation, senescence, diabetes, insulin resistance, inflammation, neurodegenerative disorders or atherosclerosis.

a clear distinction between ceramides that function in the multilamellar barrier outside the cell and ceramides that function as a second messenger inside the cell. Moreover, each mechanism is a reversible pathway, thus, S1P and ceramide-1-phosphate play opposing roles in cell growth inhibition and apoptosis.

A study reported that for keratinocytes in the epidermis, intracellular ceramides induce the apoptosis of cells exposed to UVA radiation, thereby controlling the expression of ICAM1 by mediating AP2 activity (67). Moreover, processed short-chain ceramides may permeate into cells and induce apoptosis and differentiation, and reduce proliferation by activating apoptosis signal-regulating kinase 1 (ASK-1), p38 and caspase-14 in cells (68-70). Moreover, glucosylceramide and S1P, derivatives of ceramide, also induce keratinocyte differentiation (71). Furthermore, in melanocytes, AKT 
phosphorylation is reduced by short-chain ceramide, thereby decreasing melanocyte growth and increasing melanin synthesis (72).

\section{Conclusion}

Ceramides and their derivatives form the lamellar barrier of the skin and facilitate the differentiation of keratinocytes, thereby creating the epidermal barrier. Thus, they limit the movement of material through the skin, maintain skin moisture by preventing dehydration and prevent microbes and allergens from entering tissues from the outside. As a consequence, impaired ceramide synthesis damages the barrier function of the epidermis, making it impossible for the skin to control moisture levels. External microbes and allergens may then enter the tissues and dehydrate the skin, causing inflammation and resulting in such cutaneous diseases as atopic dermatitis or psoriasis. Accordingly, it is crucial that the skin controls the type and amount of ceramides produced in the skin. Ceramides perform a number of functions inside cells, creating signals associated with apoptosis, proliferation and differentiation. The metabolism of ceramides may suppress apoptosis. Therefore, through the synthesis and metabolic conversion of ceramides, it is possible to control the apoptosis, proliferation and differentiation of skin cells and the formation of the skin barrier.

\section{Acknowledgements}

The present study was supported by the KU Research Professor Program (H.-J.C.) of Konkuk University. This study was also supported by grants from the Ministry of Science, ICT and Future Planning (grant no. 20110028646), the Korean Health Technology R\&D Project, Ministry of Health and Welfare (grant no. HN13C0075), and the Ministry of Oceans and Fisheries, Republic of Korea (grant no. OF123321).

\section{References}

1. Natarajan VT, Ganju P, Ramkumar A, Grover R and Gokhale RS Multifaceted pathways protect human skin from UV radiation. Nat Chem Biol 10: 542-551, 2014.

2. Rawlings AV and Harding CR: Moisturization and skin barrier function. Dermatol Ther (Heidelb) 17 (Suppl 1): 43-48, 2004.

3. Wertz PW: Current understanding of skin biology pertinent to skin penetration: skin biochemistry. Skin Pharmacol Physiol 26 217-226, 2013.

4. Del Rosso JQ and Levin J: Clinical relevance of maintaining the structural and functional integrity of the stratum corneum: why is it important to you? J Drugs Dermatol 10 (Suppl): s5-s12, 2011

5. Jacobi OT: About the mechanism of moisture regulation in the horny layer of the skin. Proc Sci Sect Toilet Goods Assoc 31: 22-24, 1959.

6. Blank IH: Factors which influence the water content of the stratum corneum. J Invest Dermatol 18: 433-440, 1952.

7. Elias PM: Epidermal lipids, barrier function, and desquamation. J Invest Dermatol 80 (Suppl): 44s-49s, 1983.

8. Feingold KR: Thematic review series: skin lipids. The role of epidermal lipids in cutaneous permeability barrier homeostasis. J Lipid Res 48: 2531-2546, 2007.

9. Feingold KR and Elias PM: Role of lipids in the formation and maintenance of the cutaneous permeability barrier. Biochim Biophys Acta 1841: 280-294, 2014.

10. Elias PM and Wakefield JS: Mechanisms of abnormal lamellar body secretion and the dysfunctional skin barrier in patients with atopic dermatitis. J Allergy Clin Immunol 134: 781-791.e1, 2014

11. Candi E, Schmidt R and Melino G: The cornified envelope: a model of cell death in the skin. Nat Rev Mol Cell Biol 6: 328-340, 2005.
12. van Smeden J, Janssens M, Gooris GS and Bouwstra JA: The important role of stratum corneum lipids for the cutaneous barrier function. Biochim Biophys Acta 1841: 295-313, 2014.

13. Feingold KR: The regulation and role of epidermal lipid synthesis. Adv Lipid Res 24: 57-82, 1991.

14. Coderch L, López O, de la Maza A and Parra JL: Ceramides and skin function. Am J Clin Dermatol 4: 107-129, 2003.

15. Choi MJ and Maibach HI: Role of ceramides in barrier function of healthy and diseased skin. Am J Clin Dermatol 6: 215-223, 2005.

16. Ogretmen B and Hannun YA: Biologically active sphingolipids in cancer pathogenesis and treatment. Nat Rev Cancer 4: 604-616, 2004.

17. Arana L, Gangoiti P, Ouro A, Trueba M and Gómez-Muñoz A: Ceramide and ceramide 1-phosphate in health and disease. Lipids Health Dis 9: 15, 2010.

18. Mimeault M, Hauke R and Batra SK: Recent advances on the molecular mechanisms involved in the drug resistance of cancer cells and novel targeting therapies. Clin Pharmacol Ther 83: 673-691, 2008

19. Robson KJ, Stewart ME, Michelsen S, Lazo ND and Downing DT: 6-Hydroxy-4-sphingenine in human epidermal ceramides. J Lipid Res 35: 2060-2068, 1994.

20. t'Kindt R, Jorge L, Dumont E, Couturon P, David F, Sandra P and Sandra K: Profiling and characterizing skin ceramides using reversed-phase liquid chromatography-quadrupole time-of-flight mass spectrometry. Anal Chem 84: 403-411, 2012.

21. Masukawa Y, Narita H, Shimizu E, Kondo N, Sugai Y, Oba T, Homma R, Ishikawa J, Takagi Y, Kitahara T, et al: Characterization of overall ceramide species in human stratum corneum. J Lipid Res 49: 1466-1476, 2008.

22. Pruett ST, Bushnev A, Hagedorn K, Adiga M, Haynes CA, Sullards MC, Liotta DC and Merrill AH Jr: Biodiversity of sphingoid bases ('sphingosines') and related amino alcohols. J Lipid Res 49: 1621-1639, 2008.

23. Farwanah H, Pierstorff B, Schmelzer CE, Raith K, Neubert RH, Kolter T and Sandhoff K: Separation and mass spectrometric characterization of covalently bound skin ceramides using LC/APCI-MS and Nano-ESI-MS/MS. J Chromatogr B Analyt Technol Biomed Life Sci 852: 562-570, 2007.

24. Stewart ME and Downing DT: Free sphingosines of human skin include 6-hydroxysphingosine and unusually long-chain dihydrosphingosines. J Invest Dermatol 105: 613-618, 1995.

25. Nugteren DH: The enzymic chain elongation of fatty acids by rat-liver microsomes. Biochim Biophys Acta 106: 280-290, 1965.

26. Guillou H, Zadravec D, Martin PG and Jacobsson A: The key roles of elongases and desaturases in mammalian fatty acid metabolism: insights from transgenic mice. Prog Lipid Res 49: 186-199, 2010

27. Jakobsson A, Westerberg $R$ and Jacobsson A: Fatty acid elongases in mammals: their regulation and roles in metabolism. Prog Lipid Res 45: 237-249, 2006.

28. Breiden B and Sandhoff K: The role of sphingolipid metabolism in cutaneous permeability barrier formation. Biochim Biophys Acta 1841: 441-452, 2014

29. Jennemann R, Rabionet M, Gorgas K, Epstein S, Dalpke A, Rothermel U, Bayerle A, van der Hoeven F, Imgrund S, Kirsch J, et al: Loss of ceramide synthase 3 causes lethal skin barrier disruption. Hum Mol Genet 21: 586-608, 2012.

30. Rabionet M, Gorgas $\mathrm{K}$ and Sandhoff R: Ceramide synthesis in the epidermis. Biochim Biophys Acta 1841: 422-434, 2014.

31. Marekov LN and Steinert PM: Ceramides are bound to structural proteins of the human foreskin epidermal cornified cell envelope. J Biol Chem 273: 17763-17770, 1998.

32. Wertz PW and Downing DT: Ceramides of pig epidermis: structure determination. J Lipid Res 24: 759-765, 1983.

33. Mizutani Y, Mitsutake S, Tsuji K, Kihara A and Igarashi Y: Ceramide biosynthesis in keratinocyte and its role in skin function. Biochimie 91: 784-790, 2009.

34. Eckl KM, Tidhar R, Thiele H, Oji V, Hausser I, Brodesser S, Preil ML, Onal-Akan A, Stock F, Müller D, et al: Impaired epidermal ceramide synthesis causes autosomal recessive congenital ichthyosis and reveals the importance of ceramide acyl chain length. J Invest Dermatol 133: 2202-2211, 2013.

35. Holleran WM, Takagi Y and Uchida Y: Epidermal sphingolipids: metabolism, function, and roles in skin disorders. FEBS Lett 580: 5456-5466, 2006.

36. Ebel P, Imgrund S, Vom Dorp K, Hofmann K, Maier H, Drake H, Degen J, Dörmann P, Eckhardt M, Franz T and Willecke K: Ceramide synthase 4 deficiency in mice causes lipid alterations in sebum and results in alopecia. Biochem J 461: 147-158, 2014. 
37. Wertz PW: Lipids and barrier function of the skin. Acta Derm Venereol Suppl (Stockh) 208: 7-11, 2000.

38. Farwanah H, Raith K, Neubert RH and Wohlrab J: Ceramide profiles of the uninvolved skin in atopic dermatitis and psoriasis are comparable to those of healthy skin. Arch Dermatol Res 296 514-521, 2005.

39. Imokawa G, Abe A, Jin K, Higaki Y, Kawashima M and Hidano A: Decreased level of ceramides in stratum corneum of atopic dermatitis: an etiologic factor in atopic dry skin? J Invest Dermatol 96: 523-526, 1991.

40. Houben E, Holleran WM, Yaginuma T, Mao C, Obeid LM, Rogiers V, Takagi Y, Elias PM and Uchida Y: Differentiation-associated expression of ceramidase isoforms in cultured keratinocytes and epidermis. J Lipid Res 47: 1063-1070, 2006.

41. Bouwstra JA, Gooris GS, Dubbelaar FE, Weerheim AM, Ijzerman AP and Ponec M: Role of ceramide 1 in the molecular organization of the stratum corneum lipids. J Lipid Res 39: 186-196, 1998

42. Bouwstra JA, Gooris GS, Dubbelaar FE and Ponec M: Phase behavior of stratum corneum lipid mixtures based on human ceramides: the role of natural and synthetic ceramide 1 . J Invest Dermatol 118: 606-617, 2002.

43. Hanada K: Intracellular trafficking of ceramide by ceramide transfer protein. Proc Jpn Acad Ser B Phys Biol Sci 86: 426-437, 2010.

44. Ponnusamy S, Meyers-Needham M, Senkal CE, Saddoughi SA, Sentelle D, Selvam SP, Salas A and Ogretmen B: Sphingolipids and cancer: ceramide and sphingosine-1-phosphate in the regulation of cell death and drug resistance. Future Oncol 6 : 1603-1624, 2010

45. Jensen JM, Förl M, Winoto-Morbach S, Seite S, Schunck M, Proksch E and Schütze S: Acid and neutral sphingomyelinase, ceramide synthase, and acid ceramidase activities in cutaneous aging. Exp Dermatol 14: 609-618, 2005.

46. Jensen JM, Fölster-Holst R, Baranowsky A, Schunck M, Winoto-Morbach S, Neumann C, Schütze S and Proksch E: Impaired sphingomyelinase activity and epidermal differentiation in atopic dermatitis. J Invest Dermatol 122: 1423-1431, 2004.

47. Swanbeck G and Thyresson N: A study of the state of aggregation of the lipids in normal and psoriatic horny layer. Acta Derm Venereol 42: 445-447, 1962.

48. Swanbeck G and Thyresson N: An x-ray diffraction study of scales from different dermatoses. Acta Derm Venereol 41: 289-296, 1961.

49. Breathnach AS, Goodman T, Stolinski C and Gross M Freeze-fracture replication of cells of stratum corneum of human epidermis. J Anat 114: 65-81, 1973.

50. Breathnach AS: Aspects of epidermal ultrastructure. J Invest Dermatol 65: 2-15, 1975.

51. White SH, Mirejovsky D and King GI: Structure of lamellar lipid domains and corneocyte envelopes of murine stratum corneum. An X-ray diffraction study. Biochemistry 27: 3725-3732, 1988.

52. Schreiner V, Gooris GS, Pfeiffer S, Lanzendörfer G, Wenck H, Diembeck W, Proksch E and Bouwstra J: Barrier characteristics of different human skin types investigated with X-ray diffraction, lipid analysis, and electron microscopy imaging. J Invest Dermatol 114: 654-660, 2000.

53. Moore DJ, Rerek ME and Mendelsohn R: Lipid domains and orthorhombic phases in model stratum corneum: evidence from Fourier transform infrared spectroscopy studies. Biochem Biophys Res Commun 231: 797-801, 1997.

54. Hannun YA: Functions of ceramide in coordinating cellular responses to stress. Science 274: 1855-1859, 1996.

55. Ruvolo PP: Intracellular signal transduction pathways activated by ceramide and its metabolites. Pharmacol Res 47: 383-392, 2003
56. Ballou LR, Laulederkind SJ, Rosloniec EF and Raghow R Ceramide signalling and the immune response. Biochim Biophys Acta 1301: 273-287, 1996

57. Geilen $\mathrm{CC}$, Wieder $\mathrm{T}$ and Orfanos CE: Ceramide signalling: regulatory role in cell proliferation, differentiation and apoptosis in human epidermis. Arch Dermatol Res 289: 559-566, 1997.

58. Uchida Y: Ceramide signaling in mammalian epidermis. Biochim Biophys Acta 1841: 453-462, 2014

59. Senkal CE, Ponnusamy S, Bielawski J, Hannun YA and Ogretmen B: Antiapoptotic roles of ceramide-synthase-6-generated C16-ceramide via selective regulation of the ATF6/CHOP arm of ER-stress-response pathways. FASEB J 24: 296-308, 2010.

60. Tonnetti L, Veri MC, Bonvini E and D'Adamio L: A role for neutral sphingomyelinase-mediated ceramide production in $\mathrm{T}$ cell receptor-induced apoptosis and mitogen-activated protein kinasemediated signal transduction. J Exp Med 189: 1581-1589, 1999.

61. Gómez-Muñoz A: Ceramide-1-phosphate: a novel regulator of cell activation. FEBS Lett 562: 5-10, 2004.

62. Gómez-Muñoz A, Kong JY, Salh B and Steinbrecher UP: Ceramide-1-phosphate blocks apoptosis through inhibition of acid sphingomyelinase in macrophages. J Lipid Res 45: 99-105, 2004.

63. Gomez-Muñoz A, Martin A, O'Brien L and Brindley DN: Cell-permeable ceramides inhibit the stimulation of DNA synthesis and phospholipase D activity by phosphatidate and lysophosphatidate in rat fibroblasts. J Biol Chem 269: 8937-8943, 1994.

64. Gómez-Muñoz A, Kong JY, Parhar K, Wang SW, Gangoiti P, González M,Eivemark S, Salh B, Duronio V and Steinbrecher UP: Ceramide-1-phosphate promotes cell survival through activation of the phosphatidylinositol 3-kinase/protein kinase B pathway. FEBS Lett 579: 3744-3750, 2005.

65. Bourbon NA,Sandirasegarane Land Kester M: Ceramide-induced inhibition of Akt is mediated through protein kinase Czeta: implications for growth arrest. J Biol Chem 277: 3286-3292, 2002.

66. Schubert KM, Scheid MP and Duronio V: Ceramide inhibits protein kinase $\mathrm{B} / \mathrm{Akt}$ by promoting dephosphorylation of serine 473. J Biol Chem 275: 13330-13335, 2000.

67. Grether-Beck S, Bonizzi G, Schmitt-Brenden H, Felsner I, Timmer A, Sies H, Johnson JP, Piette J and Krutmann J: Non-enzymatic triggering of the ceramide signalling cascade by solar UVA radiation. EMBO J 19: 5793-5800, 2000.

68. Wakita H, Tokura Y, Yagi H, Nishimura K, Furukawa F and Takigawa M: Keratinocyte differentiation is induced by cell-permeant ceramides and its proliferation is promoted by sphingosine. Arch Dermatol Res 286: 350-354, 1994.

69. Sayama K, Hanakawa Y, Shirakata Y, Yamasaki K, Sawada Y, Sun L, Yamanishi K, Ichijo H and Hashimoto K: Apoptosis signal-regulating kinase 1 (ASK1) is an intracellular inducer of keratinocyte differentiation. J Biol Chem 276: 999-1004, 2001.

70. Jiang YJ, Kim P, Uchida Y, Elias PM, Bikle DD, Grunfeld C and Feingold KR: Ceramides stimulate caspase-14 expression in human keratinocytes. Exp Dermatol 22: 113-118, 2013.

71. Manggau M, Kim DS, Ruwisch L, Vogler R, Korting HC, Schäfer-Korting $M$ and Kleuser $B$ : 1Alpha,25-dihydroxyvitamin D3 protects human keratinocytes from apoptosis by the formation of sphingosine-1-phosphate. J Invest Dermatol 117: 1241-1249, 2001.

72. Kim DS, Kim SY, Moon SJ, Chung JH, Kim KH, Cho KH and Park KC: Ceramide inhibits cell proliferation through Akt/PKB inactivation and decreases melanin synthesis in Mel-Ab cells. Pigment Cell Res 14: 110-115, 2001. 